\title{
Physico - Chemical Study of Transition Metal Complexes with Schiff's Base derived from Naphthaldehyde and Substituted Aromatic Amines
}

\author{
PREM MOHAN MISHRA ${ }^{1}$ and VEENA MISHRA ${ }^{2}$ \\ 1'Department of Chemistry, M.L.S.M. College, Darbhanga, India. \\ ${ }^{2}$ L.N. Mithila University, Darbhanga, Bihar - 846004, India. \\ *Corresponding author E-mail: mishrapm6@gmail.com \\ http://dx.doi.org/10.13005/ojc/320549
}

(Received: July 20, 2016; Accepted: September 22, 2016)

\begin{abstract}
In continuation of the previous work here we are going to report stability constant values of transition metals like $\mathrm{Cu}(\mathrm{II}), \mathrm{Ni}(\mathrm{II}), \mathrm{Co}(\mathrm{II})$ and $\mathrm{Zn}$ (II) with Schiff;s base ligand. Schiff's base ligands were synthesized by the condensation reaction of $\beta$-Naphthaldehyde with 7- Hydroxy naphthalene2 - amine .Nitrate salts of divalent cobalt, nickel, copper and zinc were estimated by usual methods. Ligand was analysed for elements by standard method. $\mathrm{pH}$ metric titrations were carried out with the help of digital $\mathrm{pH}$ meter and stability constant of complexes of these metals with the ligands synthesized were computed by Irving- Rossotti technique modified by Calvin-Bjerrum. The stability constant values of metals for the given ligand were found to be in the order $\mathrm{Cu}(\mathrm{II})>\mathrm{Ni}(\mathrm{II}), \mathrm{Co}(\mathrm{II})>$ $\mathrm{Zn}$ (II). This result is in agreement with the natural order proposed by Irving-William.
\end{abstract}

Keywords : Schiff's base, complex compound, Stability constant, Irving - Rossotti titration technique, Naphthaldehyde, Thermodynamic parameters.

\section{INTRODUCTION}

These days considerable attention is being paid to the chemistry of complex compounds of Schiff's base containing nitrogen and other donor atoms. ${ }^{1-4}$ Schiff's base offer a versatile and flexible series of ligands capable to bind with variety of metal ions to give complexes with varying properties. These complexes are biologically active ${ }^{5}$ and have wide potential applications in many fields such as catalysis $^{6}$, electrochemistry ${ }^{7}$ and medicines Studies ${ }^{8}$ have shown that metal complexes act as antitumour, antiviral, anti cancer ${ }^{10}$ and other many anti bacterial agents.

A large number of polydentate Schiff's base compounds have been synthesized and their complexes have been structurally characterized and extensively investigated. But little is known for their stability in aqueous solution in which it is used. Hence, the title project have been under taken. Here in the stability constant of complexes of divalent 
transition metals i.e. $\mathrm{Co}(\mathrm{II}), \mathrm{Ni}(\mathrm{II}), \mathrm{Cu}(\mathrm{II})$ and $\mathrm{Zn}(\mathrm{II})$ with Schiff's base ligands have been determined.

\section{Experimental}

Nitrate salts of divalent $\mathrm{Co}, \mathrm{Ni}, \mathrm{Cu}$ and $\mathrm{Zn}$ all were E. Merck. All other chemicals used were AnalR grade and used without further purification. Elemental analysis of metal salts were done by volumetric and gravimetric methods. Double distilled and deionised water was used throughout the experiment. All titrations were done in aqueous-dioxane medium in the ratio 3:2 (v/v). Dioxane was purified by standard method.

Schiff's base ligands were synthesized by the condensation of $\beta-N a p h t h a l d e h y d e$ with 7-Hydroxy naphthalene - $2-$ amine. $3.5 \mathrm{~g}$ of aldehyde in solution was mixed with nearly $3.0 \mathrm{~g}$ amine. The mixture was boiled under reflux in the presence of glacial acetic acid for about 2 hours. The solution was concentrated and cooled to $0^{\circ} \mathrm{C}$. The product obtained was filtered, washed several times and recrystallized from ethanol. The yield of product was nearly $2.6 \mathrm{~g}$.<smiles>O=Cc1ccc2ccccc2c1</smiles><smiles>Nc1ccc2ccc(O)cc2c1</smiles><smiles>CCCCC</smiles><smiles>Oc1ccc2ccc(/N=C/c3ccc4ccccc4c3)cc2c1</smiles>

Table 1: Concentrations used in the experiment

\begin{tabular}{lcccccc}
\hline $\begin{array}{l}\text { Metal } \\
\text { I lons }\end{array}$ & $\begin{array}{c}\text { Vo } \\
(\mathbf{m L})\end{array}$ & $\mathbf{Y}$ & $\mathbf{N}^{\mathbf{O}}$ & $\mathbf{E}^{\mathrm{O}}$ & $\mathbf{T}_{\mathrm{L}}$ & $\mathbf{T}_{M}$ \\
\hline Co (II) & 100 & 1 & $1.0(\mathrm{M})$ & $1.0 \times 10^{-2}(\mathrm{M})$ & $2.4 \times 10^{-3}(\mathrm{M})$ & $5.0 \times 10^{-4}(\mathrm{M})$ \\
$\mathrm{Ni}(\mathrm{II})$ & 100 & 1 & $1.0(\mathrm{M})$ & $1.0 \times 10^{-2}(\mathrm{M})$ & $2.4 \times 10^{-3}(\mathrm{M})$ & $5.0 \times 10^{-4}(\mathrm{M})$ \\
$\mathrm{Cu}(\mathrm{II})$ & 100 & 1 & $1.0(\mathrm{M})$ & $1.0 \times 10^{-2}(\mathrm{M})$ & $2.4 \times 10^{-3}(\mathrm{M})$ & $5.0 \times 10^{-4}(\mathrm{M})$ \\
$\mathrm{Zn}(\mathrm{II})$ & 100 & 1 & $1.0(\mathrm{M})$ & $1.0 \times 10^{-2}(\mathrm{M})$ & $2.4 \times 10^{-3}(\mathrm{M})$ & $5.0 \times 10^{-4}(\mathrm{M})$ \\
\hline
\end{tabular}

Calvin - Bjerrum $\mathrm{pH}$ metric titration of acid, acid + ligand and acid + ligand + metal ions solutions were done at constant ionic strength of $0.1 \mathrm{M} \mathrm{KNO}_{3}$ at $298 \mathrm{~K}$ temperature in an inert atmosphere of nitrogen.

The same process of titration were repeated for all the four $\mathrm{Co}, \mathrm{Ni}, \mathrm{Cu}$ and $\mathrm{Zn}$ metal ions. The change in colour and appearance of turbidity at particular $\mathrm{pH}$ value were recorded simultaneously.

The change in $\mathrm{pH}$ of the solutions with each addition of alkali was recorded in Table no. 2.

\section{RESULTS}

A graph was plotted between $\mathrm{pH}$ meter reading $[B]$ and volume of alkali added in each case,
(Figure - 1. ) Three titration curves obtained for each metal ions are acid titration curve (a), ligand titration curve (b) and complex titration curve (c) respectively.

The values of volumes $\left(\mathrm{V}_{1}, \mathrm{~V}_{2}, \& \mathrm{~V}_{3}\right)$ corresponding to the same $\mathrm{pH}$ values were read from acid, ligand and complex titration curves (a), (b) and (c) respectively obtained from the experiment at temperature $298 \mathrm{~K}$ given in Figure - 1 .

The $\bar{n}_{A}, \bar{n} \& \mathrm{P} \mathrm{L}$ are calculated using standard expressions

$\bar{n}_{A}=1+\left[\left(\mathrm{V}_{1}-\mathrm{V}_{2}\right) /\left(\mathrm{V}^{\mathrm{O}}+\mathrm{V}_{1}\right)\right]\left(\mathrm{N}^{\mathrm{O}}+\mathrm{E}^{\circ}\right) / \mathrm{T} \underset{L}{o}$

$\bar{n}=\left[\left(\mathrm{V}_{3}-\mathrm{V}_{2}\right) /\left(\mathrm{V}^{\mathrm{O}}+\mathrm{V}_{1}\right)\right]\left[\left(\mathrm{N}^{\mathrm{O}}+\mathrm{E}^{\mathrm{O}}\right) / \mathrm{T}\right] \times 1 / \bar{n}_{A}$ 
Table 2: Volume of alkali consumed in different titrations

\begin{tabular}{|c|c|c|c|c|c|c|}
\hline \multicolumn{3}{|c|}{$\begin{array}{l}\text { Ligand - } \mathrm{HNNCl}\left(\mathrm{L}_{1}\right) \\
\mu^{\circ}=0.10(\mathrm{M}) \mathrm{KNO}_{3}\end{array}$} & \multicolumn{3}{|c|}{$\begin{array}{l}\text { Temp. } 298 \pm 1 \mathrm{~K} \\
\text { Water : dioxane medium }(\mathrm{v} / \mathrm{v})=3: 2\end{array}$} & \multirow[b]{3}{*}{$\mathrm{H}^{+}+\mathrm{L}+\mathrm{Zn}(\mathrm{II})$} \\
\hline \multirow[b]{2}{*}{$\begin{array}{l}\text { Vol. of alkali } \\
\text { added in } \mathrm{mL}\end{array}$} & \multirow[b]{2}{*}{$\mathrm{H}^{+}$} & \multirow[b]{2}{*}{$\mathrm{H}^{+}+\mathrm{L}$} & \multirow[b]{2}{*}{$\mathrm{H}^{+}+\mathrm{L}+\mathrm{Co}(\mathrm{II})$} & \multicolumn{2}{|c|}{$\mathrm{pH}$ - meter reading $(\mathrm{B})$} & \\
\hline & & & & $\mathrm{H}^{+}+\mathrm{L}+\mathrm{Ni}(\mathrm{II})$ & $\mathrm{H}^{+}+\mathrm{L}+\mathrm{Cu}(\mathrm{II})$ & \\
\hline 0.0 & 5.05 & 5.35 & 5.32 & 5.3 & 5.3 & 5.3 \\
\hline 0.1 & 5.15 & 5.43 & 5.4 & 5.42 & 5.42 & 5.44 \\
\hline 0.2 & 5.33 & 5.57 & 5.52 & 5.55 & 5.5 & 5.52 \\
\hline 0.3 & 5.53 & 5.87 & 5.8 & 5.86 & 5.82 & 5.82 \\
\hline 0.4 & 5.95 & 5.91 & 6.32 & 6.32 & 6.32 & 6.42 \\
\hline 0.5 & 6.13 & 6.73 & 6.5 & 6.52 & 6.52 & 6.5 \\
\hline 0.6 & 6.45 & 6.9 & 6.72 & 6.72 & 6.7 & 6.74 \\
\hline 0.7 & 7.9 & 7.15 & 7.1 & 7.12 & 7.1 & 7.12 \\
\hline 0.8 & 8.9 & 7.75 & 7.72 & 7.79 & 7.82 & 7.74 \\
\hline 0.9 & 10.15 & 10.27 & 8.55 & 8.98 & 8.5 & 8.62 \\
\hline 1.0 & 11.9 & 11.1 & 10.5 & 10.75 & 9.22 & 9.12 \\
\hline 1.1 & 12.85 & 12.43 & 11.25 & 11.75 & 9.62 & 10.34 \\
\hline 1.2 & 13.15 & 12.57 & 11.70 & 12.10 & 10.20 & 10.56 \\
\hline
\end{tabular}

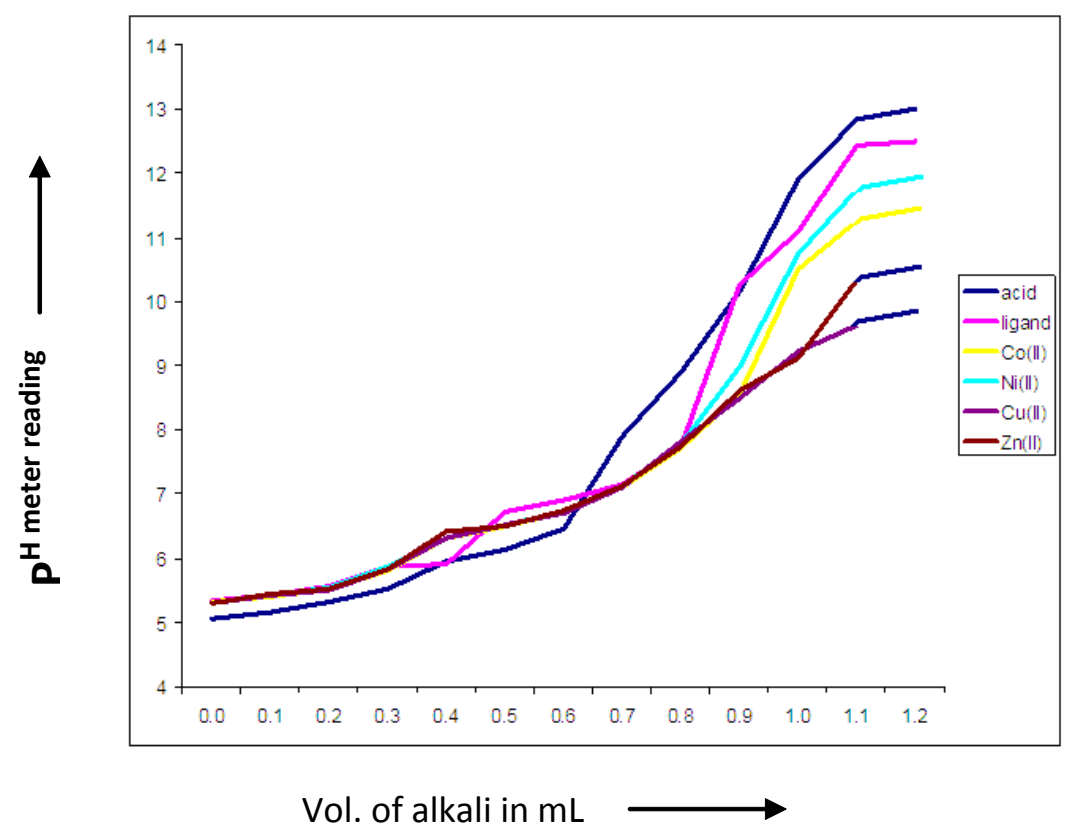

Temp. $298 \pm 1 \mathrm{~K}$ $\mu^{\circ}=0.10(\mathrm{M}) \mathrm{KNO}_{3}$ Water : dioxane $=3: 2(\mathrm{v} / \mathrm{v})$

Fig. 1: Experimental curve with ligand $\mathrm{HNNCI}$ 
$\mathrm{P} \mathrm{L}=\log \left[\sum_{j=0}^{j} \beta_{j}^{0} H(1 / \operatorname{anti} \log \mathrm{B})\left(\mathrm{V}^{\mathrm{O}}+\mathrm{V}_{3}\right)\left(T_{L}^{O}-\bar{n} T_{M}^{o}\right) V^{0}\right]$

\section{Proton ligand stability constant}

The ligand titration curve is above the acid titration curve showing the basic nature of ligand and it is well separated from the acid titration curve at $\mathrm{pH}=6.3$ at temp $298 \mathrm{~K}$. The ligand curves run parallel

Table $3:$ The values of $\bar{n}_{A}$ at various $\mathrm{pH}$ reading $[\mathrm{B}]$

\begin{tabular}{|c|c|c|c|}
\hline \multicolumn{2}{|c|}{$\begin{array}{l}\text { Ligand }-\mathrm{HNNCl} \\
\mu^{\circ}=0.10(\mathrm{M}) \mathrm{KNO}_{3}\end{array}$} & \multicolumn{2}{|c|}{$\begin{array}{l}\text { Temp.: } 298 \pm 1 \mathrm{~K} \\
\text { Water : dioxane }=3: 2(\mathrm{v} / \mathrm{v})\end{array}$} \\
\hline [B] & $V_{2}-V_{1}$ & & $\log \bar{n}_{A} /\left(1-\bar{n}_{A}\right)$ \\
\hline 5.2 & 0.005 & 0.8878 & \\
\hline 5.4 & 0.007 & 0.8840 & \\
\hline 5.6 & 0.007 & 0.8832 & \\
\hline 5.8 & 0.008 & 0.8812 & \\
\hline 6.0 & 0.008 & 0.8814 & \\
\hline 6.2 & 0.009 & 0.8742 & \\
\hline 6.4 & 0.009 & 0.8724 & \\
\hline 6.6 & 0.010 & 0.8682 & \\
\hline 6.8 & 0.014 & 0.8610 & \\
\hline 7.0 & 0.014 & 0.8442 & \\
\hline 7.2 & 0.014 & 0.8362 & \\
\hline 7.4 & 0.015 & 0.8360 & 1.2642 \\
\hline 7.6 & 0.017 & 0.8282 & 1.2090 \\
\hline 7.8 & 0.017 & 0.8196 & 1.0402 \\
\hline 8.0 & 0.018 & 0.8121 & 1.0252 \\
\hline 8.2 & 0.022 & 0.7986 & 0.8530 \\
\hline 8.4 & 0.024 & 0.7964 & 0.7942 \\
\hline 8.6 & 0.032 & 0.7882 & 0.7020 \\
\hline 8.8 & 0.034 & 0.7834 & 0.6320 \\
\hline 9.0 & 0.040 & 0.7602 & 0.4582 \\
\hline 9.2 & 0.052 & 0.7120 & 0.3904 \\
\hline 9.4 & 0.054 & 0.6794 & 0.3490 \\
\hline 9.6 & 0.060 & 0.6555 & 0.2904 \\
\hline 9.8 & 0.062 & 0.6274 & 0.2272 \\
\hline 10.0 & 0.070 & 0.5996 & 0.4672 \\
\hline 10.2 & 0.084 & 0.5674 & 0.4032 \\
\hline 10.4 & 0.092 & 0.5322 & 0.3344 \\
\hline 10.6 & 0.102 & 0.4883 & 0.2530 \\
\hline 10.8 & 0.110 & 0.4782 & 0.0492 \\
\hline 11.0 & 0.122 & 0.4672 & 0.0310 \\
\hline 11.2 & 0.142 & 0.4394 & -0.2050 \\
\hline
\end{tabular}

to the acid titration curve indicating the smooth dissociation of the ligand.

The values of $\bar{n}_{A}$ at various $\mathrm{pH}$ reading [B] was calculated from the acid and ligand titration curves and recorded in table 3 .

The formation curve obtained from the plot of $\bar{n}_{A}$ vs [B] extends from 0.43 to 0.88 (Figure 2 ) at temp $298 \mathrm{~K}$.

The dissociation of ligand may be represented as

$$
\mathrm{HL} \rightleftharpoons \mathrm{H}^{+}+\mathrm{L}^{-}
$$

The value of proton ligand stability constant was calculated by half integral method and it was further corroborated by linear plot method. $\left(\log \bar{n}_{A} /\left(1-\bar{n}_{A}\right)\right.$ vs [B] Figure. -3.

\section{Table 4: Values of $\bar{n}$ and $\mathrm{P}^{\mathrm{L}}$ at various [B] values}

\begin{tabular}{cccc}
\hline $\begin{array}{l}\text { Co }(\mathrm{II})+\mathrm{HNNCl} \\
\mu^{\circ}=0.10(\mathrm{M}) \mathrm{KNO}_{3}\end{array}$ & \multicolumn{2}{c}{ Temp: $298 \pm 1 \mathrm{~K}$} \\
\hline [B] & $\mathbf{V}_{3}-\mathrm{V}_{2}$ & $\bar{n}$ & $\mathrm{PL}$ \\
\hline 5.0 & 0.004 & 0.2222 & 8.1650 \\
5.2 & 0.006 & 0.3244 & 7.9762 \\
5.4 & 0.010 & 0.4265 & 7.7862 \\
5.6 & 0.018 & 0.5914 & 7.6014 \\
5.8 & 0.032 & 0.5764 & 7.4124 \\
6.0 & 0.042 & 0.7870 & 7.2412 \\
6.2 & 0.052 & 1.8662 & 7.0634 \\
6.4 & 0.060 & 1.2842 & 6.8858 \\
6.6 & 0.072 & 1.58404 & 6.7092 \\
6.8 & 0.074 & 1.7964 & 6.5364 \\
7.0 & 0.090 & 1.8682 & 6.3742 \\
\hline
\end{tabular}

Table 5 : Values of $\mathrm{P}^{\mathrm{L}}$ at various values of $\log \bar{n} /(1-\bar{n})$ and $\log (2-\bar{n} /(\bar{n}-1)$

\begin{tabular}{lccc}
\hline $\begin{array}{l}\text { Co }(\mathrm{II})+\mathrm{HNNCl} \\
\mu^{\circ}=0.10(\mathrm{M}) \mathrm{KNO}_{3}\end{array}$ & \multicolumn{2}{l}{ Temp : $298 \quad 1 \mathrm{~K}$} \\
& Water: Dioxane $=3: 2(\mathrm{v} / \mathrm{v})$ \\
\hline $\log \bar{n} /(1-\bar{n})$ & $\mathrm{PL}$ & $\log (\mathbf{2}-\bar{n} /(\bar{n}-\mathbf{1})$ & $\mathrm{PL}$ \\
\hline-0.9560 & 8.9754 & 0.0262 & 7.7092 \\
-0.4134 & 8.7850 & -0.3622 & 7.5364 \\
0.4212 & 8.4132 & & \\
0.7904 & 8.2402 & & \\
\hline
\end{tabular}




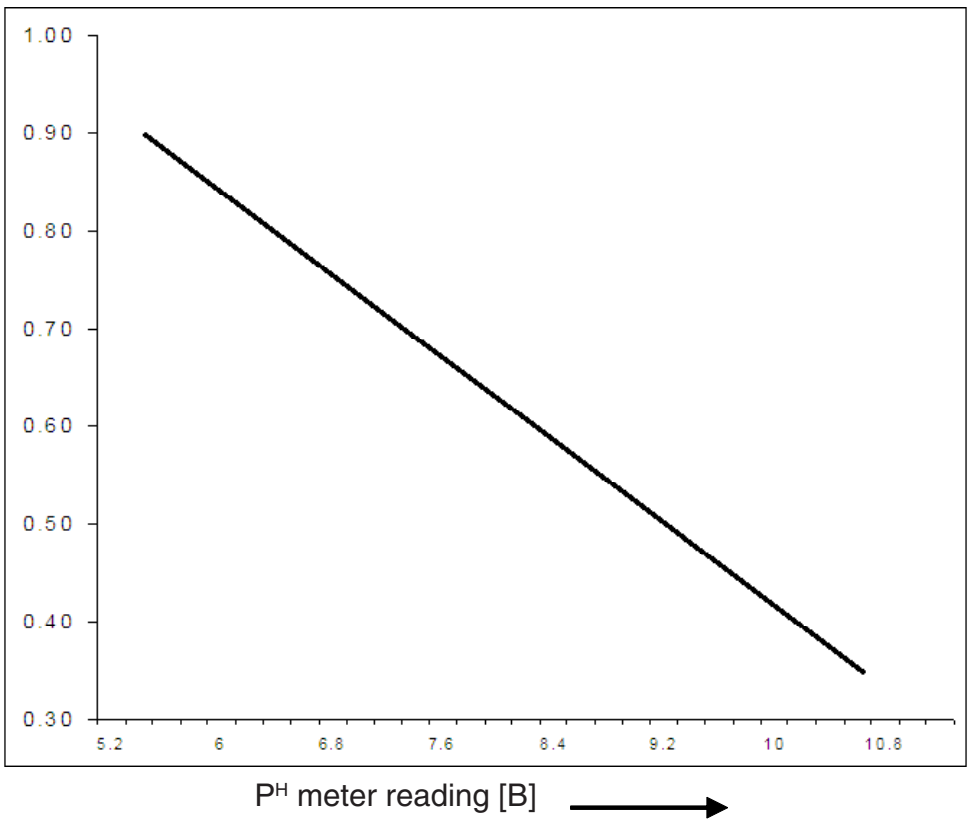

Temp. $298 \pm 1 \mathrm{~K}$ $\mu^{\circ}=0.10(\mathrm{M}) \mathrm{KNO}_{3}$

Water: Dioxane $=3: 2(\mathrm{v} / \mathrm{v})$

Fig. 2: Formation curve of ligand - HNNCI Plot of $n_{A}$ Vs [B]

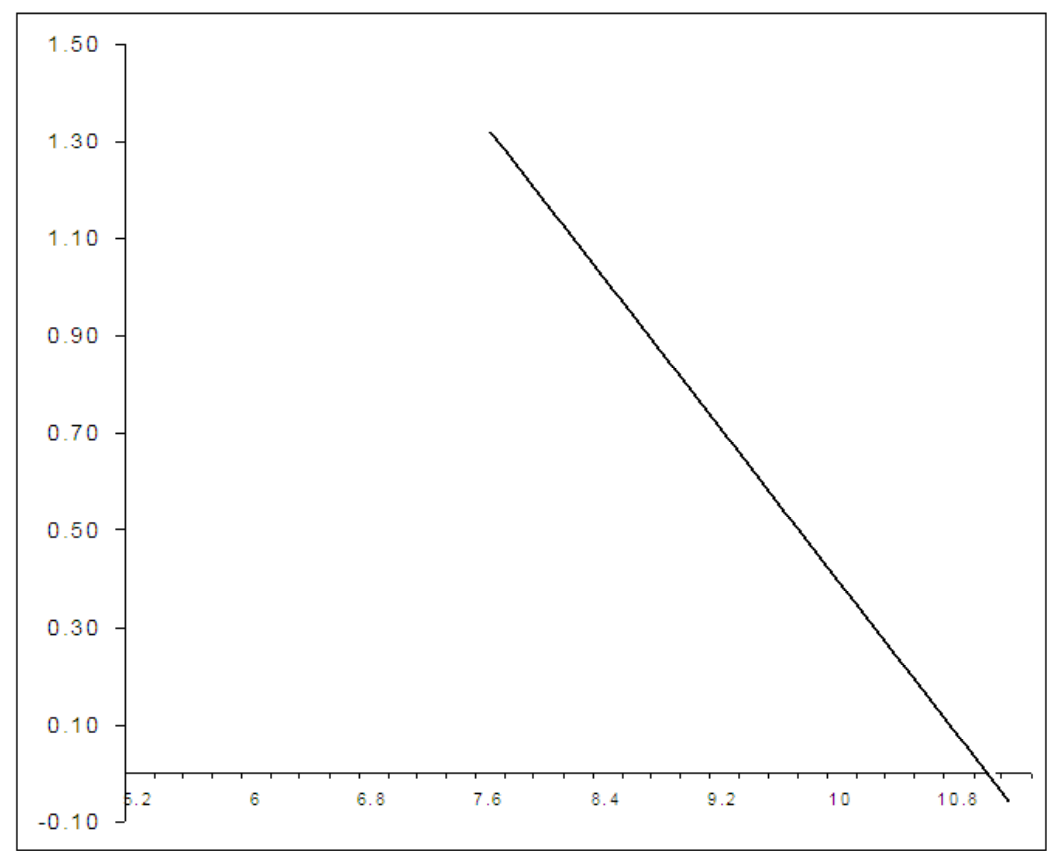

Ligand ; $\mathrm{HNNCl}$ $\mu^{\circ}=0.10(\mathrm{M}) \mathrm{KNO}_{3}$
Temp : $298 \pm 1 \mathrm{~K}$

Water: Dioxane $=3: 2(\mathrm{v} / \mathrm{v})[\mathrm{B}]$

Fig. 3: Linear plot of $\log \left(\log \bar{n}_{A} /(1\right.$ - ) Vs [B] 
The complex titration curve of the system crossed the ligand mixture curve at $\mathrm{pH} 5.35$ for $\mathrm{Co}(\mathrm{II}), \mathrm{pH} 4.88$ for $\mathrm{Ni}(\mathrm{II})$, at $\mathrm{pH}=5.90$ for $\mathrm{Cu}(\mathrm{II})$ and $\mathrm{pH} 5.68$ for $\mathrm{Zn}(\mathrm{II})$ - ligand system indicating the start of complexation.

Metal titration curve run parallel to the ligand titration curve indicating the liberation of extra proton due to hydrolysis of metal ions.

Table 6: Values of $\bar{n}$ and $\mathrm{PL}$ at various $[B]$ values

\begin{tabular}{lccc}
\hline $\begin{array}{l}\mathrm{Ni}(\mathrm{II})+\mathrm{HNNCl} \\
\mu^{\circ}=0.10(\mathrm{M}) \mathrm{KNO}_{3}\end{array}$ & \multicolumn{2}{c}{ Temp : 298 $\pm 1 \mathrm{~K}$} \\
\hline $\mathrm{B}]$ & $\mathbf{V}_{3}-\mathbf{V}_{2}$ & $\bar{n}$ & $\mathrm{PL}$ \\
\hline 5.0 & 0.004 & 0.0406 & 8.1590 \\
5.2 & 0.006 & 0.1012 & 7.9644 \\
5.4 & 0.008 & 0.2454 & 7.7764 \\
5.6 & 0.014 & 0.4082 & 7.5930 \\
5.8 & 0.022 & 0.6150 & 7.4124 \\
6.0 & 0.034 & 0.8036 & 7.2322 \\
6.2 & 0.042 & 0.9932 & 7.0520 \\
6.4 & 0.052 & 1.2306 & 6.8786 \\
6.6 & 0.062 & 1.4634 & 6.7068 \\
6.8 & 0.086 & 1.6972 & 6.5368 \\
7.0 & 0.094 & 1.9684 & 6.3734 \\
\hline
\end{tabular}

Table 8 : Values of $\bar{n}$ and $\mathrm{P}^{\mathrm{L}}$ at various $[B]$ values

\begin{tabular}{lccc}
\hline $\begin{array}{l}\mathrm{Cu}(\mathrm{II})+\mathrm{HNNCl} \\
\mu^{\circ}=0.10(\mathrm{M}) \mathrm{KNO}_{3}\end{array}$ & \multicolumn{2}{c}{ Temperature $298 \pm \mathrm{K}$} \\
\hline $\mathrm{B}]$ & $\mathrm{V}_{3}-\mathrm{V}_{2}$ & $\bar{n}$ & $\mathrm{P}^{\mathrm{L}}$ \\
\hline 6.2 & 0.006 & 0.0814 & 8.5624 \\
6.4 & 0.010 & 0.1640 & 8.3702 \\
6.6 & 0.012 & 0.2672 & 8.1794 \\
6.8 & 0.014 & 0.3722 & 7.9890 \\
7.0 & 0.020 & 0.4796 & 7.7994 \\
7.2 & 0.024 & 0.6150 & 7.6124 \\
7.4 & 0.030 & 0.7632 & 7.4270 \\
7.6 & 0.042 & 0.9412 & 7.2462 \\
7.8 & 0.052 & 1.1434 & 7.0684 \\
8.0 & 0.060 & 1.3270 & 6.8912 \\
8.2 & 0.064 & 1.5364 & 6.7152 \\
8.4 & 0.080 & 1.8240 & 6.5526 \\
\hline
\end{tabular}

\section{In, Co (II) System}

Precipitation was observed at $\mathrm{pH}$ 8.9. Hence in the calculation of $\bar{n}$ only the lower $\mathrm{pH}$ region of titration curve were used.

\section{In $\mathrm{Ni}(\mathrm{II})$ System}

The curve increased regularly up to $\mathrm{pH}$ 7.56 indicating constant rate of release of proton

Table 7: Values of $\mathrm{P}^{\mathrm{L}}$ at various values of $\log n /(1-\bar{n})$ and $\log (2-\bar{n} /(\bar{n}-1)$

\begin{tabular}{ll}
\hline $\mathrm{Ni}(\mathrm{II})+\mathrm{HNNCl}$ & Temp : $298 \pm 1 \mathrm{~K}$ \\
$\mu^{\circ}=0.10(\mathrm{M}) \mathrm{KNO}_{3}$ & Water: Dioxane = 3:2(v/v)
\end{tabular}

\begin{tabular}{lccc}
\hline $\log n /(1-n)$ & $\mathbf{P}_{\mathrm{L}}$ & $\begin{array}{c}\log (2-n) / \\
(n-1)\end{array}$ & $\mathrm{PL}$ \\
\hline-0.9454 & 8.9640 & 0.6226 & 7.8782 \\
-0.4882 & 8.7770 & 0.5626 & 7.7064 \\
-0.1606 & 8.5924 & 0.4622 & 7.5362 \\
0.2040 & 8.4124 & 0.3216 & 7.4832 \\
0.6114 & 8.2322 & 0.2182 & 7.3218 \\
\hline
\end{tabular}

.No turbidity appears, which indicates that hydrolysis does not take place.

\section{In Cu(II) System}

Similarly in $\mathrm{Cu}(\mathrm{II})$ system the curve increased regularly up to $\mathrm{pH} 9.356$ indicating constant rate of release of proton .No turbidity appears, indicating that hydrolysis does not take place

Table 9 : Values of $P^{\mathrm{L}}$ at various values of $\log \bar{n} /(1-\bar{n})$ and $\log (2-\bar{n} /(\bar{n}-1)$

\begin{tabular}{|c|c|c|c|}
\hline \multicolumn{2}{|c|}{$\begin{array}{l}\mathrm{Cu}(\mathrm{II})+\mathrm{HNNCl} \\
\mu^{\circ}=0.10(\mathrm{M}) \mathrm{KNO}_{3}\end{array}$} & \multicolumn{2}{|c|}{$\begin{array}{l}\text { Temperature } 298 \pm \mathrm{K} \\
\text { Water: Dioxane }=3: 2(\mathrm{v} / \mathrm{v})\end{array}$} \\
\hline $\log \bar{n} /(1-\bar{n}$ & ) $P^{L}$ & $\begin{array}{c}\log (2-\bar{n}) / \\
(n-1)\end{array}$ & $\mathbf{P L}^{\mathrm{L}}$ \\
\hline-0.7056 & 8.3704 & 0.7752 & 7.0684 \\
\hline-0.4360 & 8.1796 & 0.3114 & 6.8902 \\
\hline-0.2262 & 7.9892 & -0.0642 & 6.7152 \\
\hline-0.0353 & 7.7994 & -0.6710 & 6.5364 \\
\hline 0.2044 & 7.6130 & -0.7128 & 6.3281 \\
\hline 0.5090 & 7.4284 & -0.8214 & 5.9216 \\
\hline
\end{tabular}


In, Zn(II) system

In case of $\mathrm{Zn}(\mathrm{II})$ system complex titration curve diverges at higher $\mathrm{pH}$ which indicates incomplete dissociation of ligand. Therefore for the

Table 10 : Values of $\bar{n}$ and $\mathrm{P}^{\mathrm{L}}$ at various $[\mathrm{B}]$ values

\begin{tabular}{lccc}
$\begin{array}{l}\text { Zn }(\mathrm{II})+\mathrm{HNNCl} \\
\mu^{\circ}=0.10(\mathrm{M}) \mathrm{KNO}_{3}\end{array}$ & \multicolumn{2}{l}{ Temp: $298 \pm 1 \mathrm{~K}$} \\
\hline $\mathbf{B}$ & $\mathbf{V}_{3}-\mathbf{V}_{2}$ & $\bar{n}$ & $\mathbf{P L}$ \\
\hline 6.0 & 0.006 & 0.1226 & 7.3646 \\
6.2 & 0.018 & 0.1852 & 7.1726 \\
6.4 & 0.010 & 0.2914 & 6.9824 \\
6.6 & 0.012 & 0.3750 & 6.7882 \\
6.8 & 0.020 & 0.5092 & 6.6032 \\
7.0 & 0.024 & 0.6366 & 6.4162 \\
7.2 & 0.030 & 0.8344 & 6.2350 \\
7.4 & 0.042 & 1.0352 & 6.0564 \\
7.6 & 0.052 & 1.2612 & 5.8832 \\
7.8 & 0.076 & 1.6668 & 5.7292 \\
8.0 & 0.084 & 1.7215 & 5.5264 \\
\hline
\end{tabular}

Table 12 : Values of protonation constant of ligand and stepwise stability constant of complexes of $\mathrm{Co}(\mathrm{II}), \mathrm{Ni}(\mathrm{II}), \mathrm{Cu}(\mathrm{II})$ and $\mathrm{Zn}$ (II) with ligand $\mathrm{HNNCl}$

\begin{tabular}{|c|c|c|c|}
\hline \multirow{2}{*}{$\begin{array}{l}\text { System } \\
\text { Metal ions }\end{array}$} & \multirow[t]{2}{*}{ Methods } & \multicolumn{2}{|c|}{ Ligand $\mathrm{HNNCl}$} \\
\hline & & $\log K_{1}$ & $\log \mathrm{K}_{2}$ \\
\hline \multirow[t]{3}{*}{$\operatorname{HNNCI}\left(\mathrm{L}_{1}\right)$} & $A$ & 10.96 & \\
\hline & $b$ & - & \\
\hline & $\mathrm{C}$ & 10.96 & \\
\hline \multirow[t]{3}{*}{ Co (II) } & $A$ & 7.56 & 6.64 \\
\hline & $b$ & 7.58 & 6.66 \\
\hline & $\mathrm{C}$ & 7.62 & 6.68 \\
\hline \multirow[t]{3}{*}{$\mathrm{Ni}(\mathrm{II})$} & $A$ & 7.52 & 6.66 \\
\hline & $b$ & 7.44 & 6.62 \\
\hline & $\mathrm{C}$ & 7.46 & 6.68 \\
\hline \multirow[t]{3}{*}{$\mathrm{Cu}$ (II) } & $A$ & 6.76 & 5.76 \\
\hline & $b$ & 6.64 & 5.04 \\
\hline & $\mathrm{c}$ & 6.84 & 5.78 \\
\hline \multirow[t]{3}{*}{$\mathrm{Zn}(\mathrm{II})$} & $A$ & 6.62 & 5.76 \\
\hline & $b$ & 6.54 & 5.72 \\
\hline & $\mathrm{C}$ & 6.68 & 5.86 \\
\hline
\end{tabular}

calculation of $\bar{n}$ only symmetrical region of the curve was used.

The value of $\bar{n}$ calculated for these metals

are

$\begin{array}{lll}\mathrm{Co}(\mathrm{II}) & - & 0.22 \text { to } 1.86 \\ \mathrm{Ni}(\mathrm{II}) & - & 0.40 \text { to } 1.96 \\ \mathrm{Cu}(\mathrm{II}) & - & 0.081 \text { to } 1.82 \\ \mathrm{Zn}(\mathrm{II}) & - & 0.12 \text { to } 1.72\end{array}$

As $\bar{n}$ value did not go beyond 2 for any of the metal indicating the formation of $\mathrm{ML}$ and $\mathrm{ML}_{2}$ type of complexes.

From the formation curve of $\bar{n}$ vs $\mathrm{P}^{\mathrm{L}}$ (Figure no.-4a,4b,4cand 4d) the values of $\log \mathrm{K}_{1}$ and $\log \mathrm{K}_{2}$ were calculated in each case. It was further corroborated

Table 11: Values of $\mathrm{P} \mathrm{L}$ at various values of $\log \bar{n} /(1-\bar{n})$ and $\log (2-\bar{n} /(\bar{n}-1)$

\begin{tabular}{llcc}
\hline $\begin{array}{l}\mathrm{Zn}(\mathrm{II})+\mathrm{HNNCl} \\
\mu^{\circ}=0.10(\mathrm{M}) \mathrm{KNO}_{3}\end{array}$ & $\begin{array}{c}\text { Temp: } 298 \pm 1 \mathrm{~K} \\
\text { Water: Dioxane }=3: 2(\mathrm{v} / \mathrm{v})\end{array}$ \\
\hline $\log \bar{n} /(\mathbf{1 -} \bar{n})$ & $\mathbf{P L}$ & $\begin{array}{c}\log (\mathbf{2}-\bar{n}) / \\
(n-1)\end{array}$ & $\mathbf{P L}$ \\
& & & \\
\hline-0.6424 & 8.1722 & 0.4526 & 6.8822 \\
-0.3860 & 7.9816 & -0.2626 & 6.7296 \\
-0.2212 & 7.6896 & -0.2012 & 6.5812 \\
0.2430 & 7.3152 & -0.1628 & 6.3158 \\
0.7024 & 7.0125 & -0.0831 & 6.2817 \\
\hline
\end{tabular}

Table 13 : Stepwise and over all stability constant of complex compounds of various metals with ligand $\mathrm{HNNCl}$ at temperature 298K

Water - Dioxane medium (v/v) $=3: 2$ $\mu^{\circ}=0.10(\mathrm{M}) \mathrm{KNO}_{3}$

\begin{tabular}{|c|c|c|c|}
\hline \multirow[t]{2}{*}{ System } & \multicolumn{3}{|c|}{ Ligand- $\mathrm{MNNCl}\left(\mathrm{L}_{1}\right)$} \\
\hline & $\log K_{1}$ & $\log K_{2}$ & $\log$ \\
\hline $\mathrm{HNNCl}\left(\mathrm{L}_{1}\right)$ & 10.98 & - & 10.98 \\
\hline Co (II) & 6.76 & 5.70 & 12.46 \\
\hline $\mathrm{Ni}$ (II) & 7.48 & 6.68 & 14.10 \\
\hline $\mathrm{Cu}(\mathrm{II})$ & 7.58 & 6.66 & 14.20 \\
\hline $\mathrm{Zn}(\mathrm{II})$ & 6.60 & 5.78 & 12.34 \\
\hline
\end{tabular}


by mid point calculation method and linear plot of $\log \frac{\bar{n}}{1-\bar{n}}$ vs $\mathrm{P}^{\mathrm{L}}$ (Figure no.- 5a, 5b, $5 c$ and $5 d$ ) and also by plot of $\log \frac{2-\bar{n}}{\bar{n}-1}$ vs

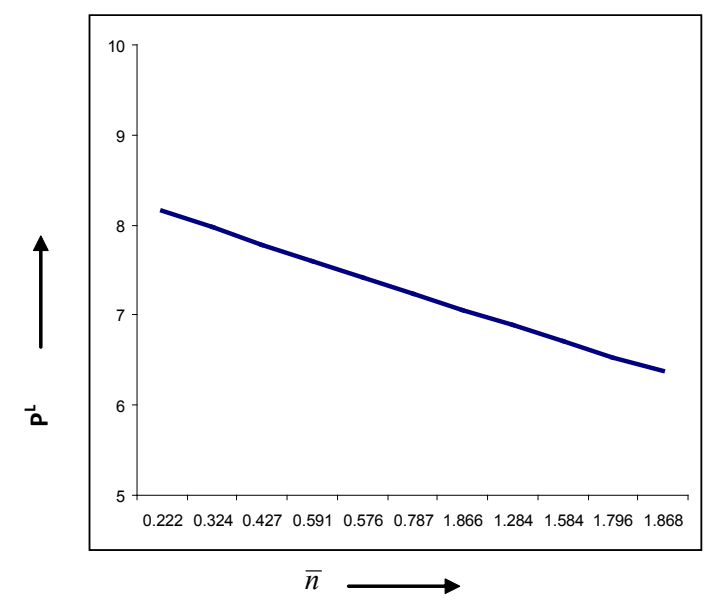

Ligand: $\mathrm{HNNCl}$

Temp. $298 \pm 1 \mathrm{~K}$

$\mu^{\circ}=0.10(\mathrm{M}) \mathrm{KNO}_{3} \quad$ Water: Dioxane $=3: 2(\mathrm{v} / \mathrm{v})$

Fig. 4a: Formation curve of Co(II) Plot of $n$ Vs $\mathrm{P}^{\mathrm{L}}$

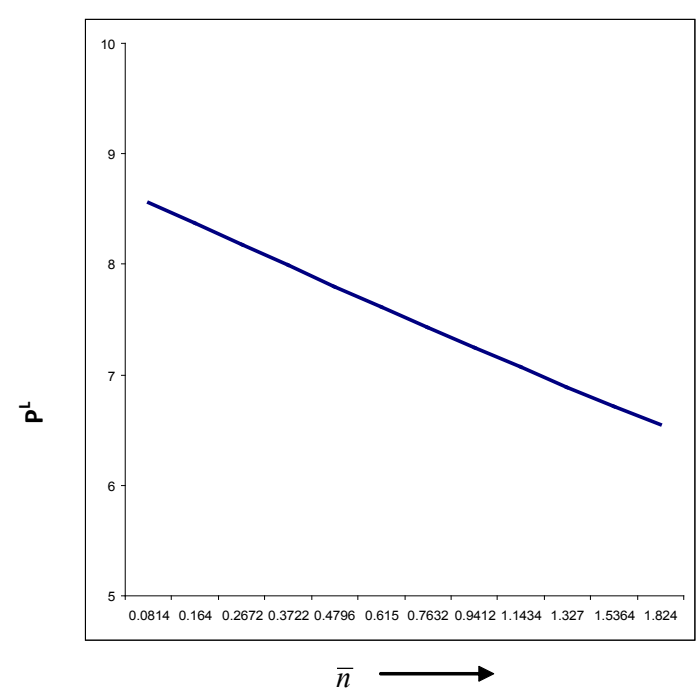

Ligand: $\mathrm{HNNCl}\left(\mathrm{L}_{1}\right) \quad$ Temp. $298 \pm 1 \mathrm{~K}$ $\mu^{\circ}=0.10(\mathrm{M}) \mathrm{KNO}_{3} \quad$ Water: Dioxane $=3: 2(\mathrm{v} / \mathrm{v})$

Fig. 4c: Formation curve of $\mathrm{Cu}$ (II) Plot of $\bar{n}$ Vs PL
$\mathrm{P}^{\mathrm{L}}$ (Graph no.-6a, 6b, 6c and 6d) at temperature 298 K.

The values of protonation constant and stepwise stability constant obtained by different

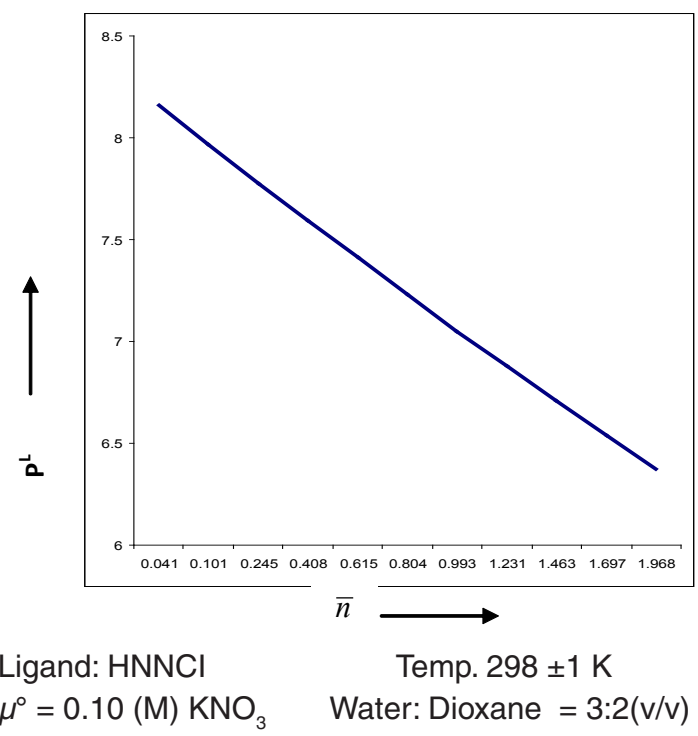

Fig.4b: Formation curve of $\mathrm{Ni}(\mathrm{II})$ Plot of $\bar{n} \mathrm{Vs} \mathrm{P}^{\mathrm{L}}$

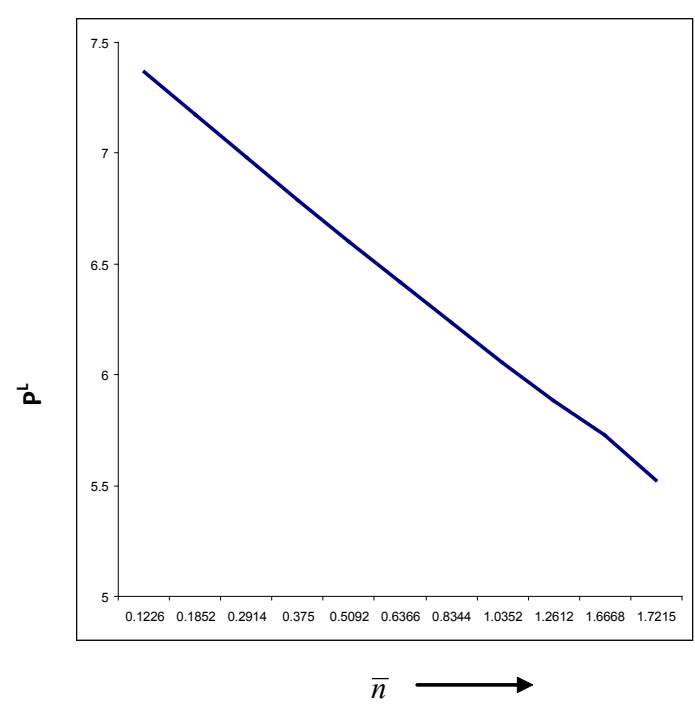

Ligand: $\mathrm{HNNCI}\left(\mathrm{L}_{1}\right)$

Temp. $298 \pm 1 \mathrm{~K}$

$\mu^{\circ}=0.10(\mathrm{M}) \mathrm{KNO}_{3} \quad$ Water: Dioxane $=3: 2(\mathrm{v} / \mathrm{v})$.

Fig.4d: Formation curve of $\mathrm{Zn}$ (II) Plot of $\bar{n} \mathrm{Vs} \mathrm{PL}^{\mathrm{L}}$ 


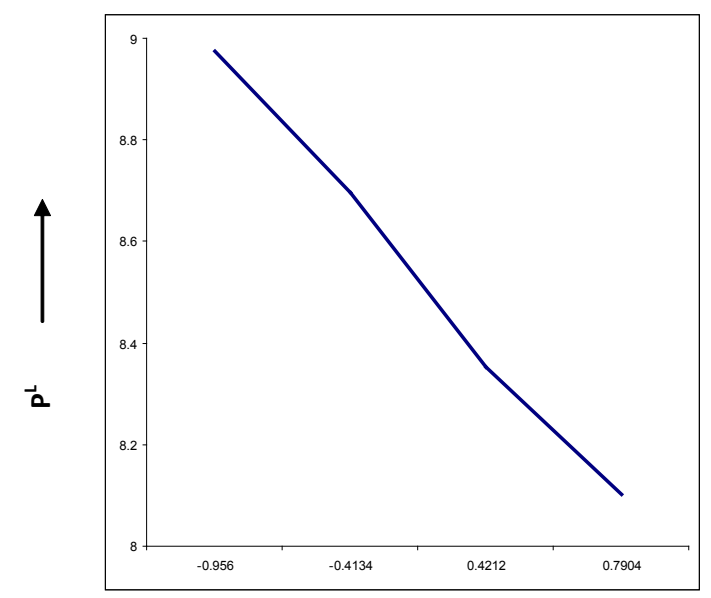

Ligand: $\mathrm{HNNCl}$ $\mu^{\circ}=0.10(\mathrm{M}) \mathrm{KNO}_{3}$

Temp. $298 \pm 1 \mathrm{~K}$

Water: Dioxane $=3: 2(\mathrm{v} / \mathrm{v})$

Fig.5a: Formation curve of $\mathrm{Co}(\mathrm{II})$

Plot of $\log (\bar{n} / 1-\bar{n}$ Vs PL

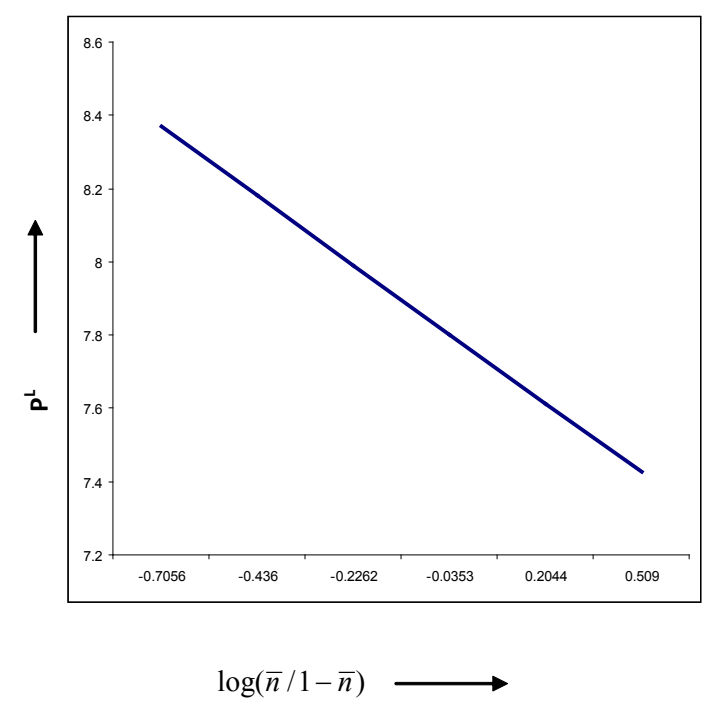

Ligand: $\mathrm{HNNCl}\left(\mathrm{L}_{1}\right) \quad$ Temp. $298 \pm 1 \mathrm{~K}$ $\mu^{\circ}=0.10(\mathrm{M}) \mathrm{KNO}_{3} \quad$ Water: Dioxane $=3: 2(\mathrm{v} / \mathrm{v})$

Fig.5c: Formation curve of Cu(II)Plot of log $(\bar{n} / 1-\bar{n})$ Vs PL

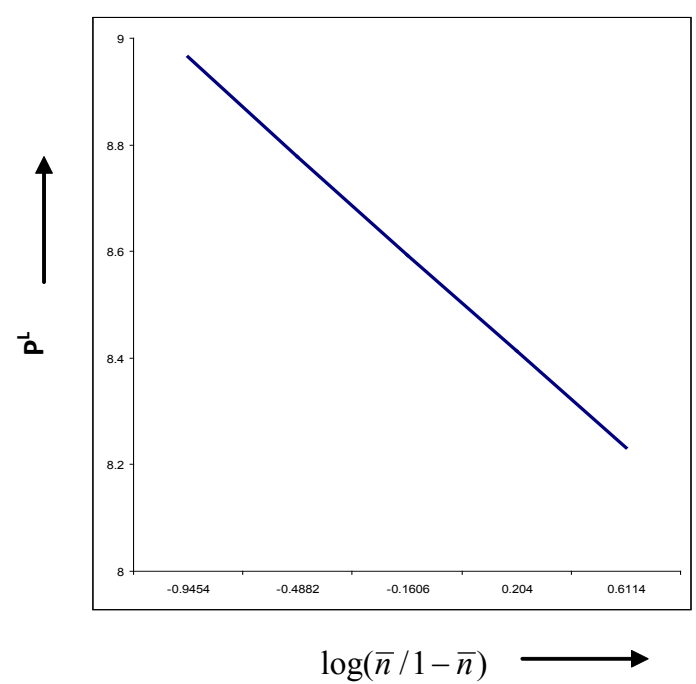

Ligand: $\mathrm{HNNCl} \quad$ Temp. $298 \pm 1 \mathrm{~K}$ $\mu^{\circ}=0.10(\mathrm{M}) \mathrm{KNO}_{3} \quad$ Water: Dioxane $=3: 2(\mathrm{v} / \mathrm{v})$

Fig. 5b: Formation curve of $\mathrm{Ni}(\mathrm{II})$ Plot of $\log (\bar{n} / 1-\bar{n}) \mathrm{Vs} \mathrm{P}^{\mathrm{L}}$

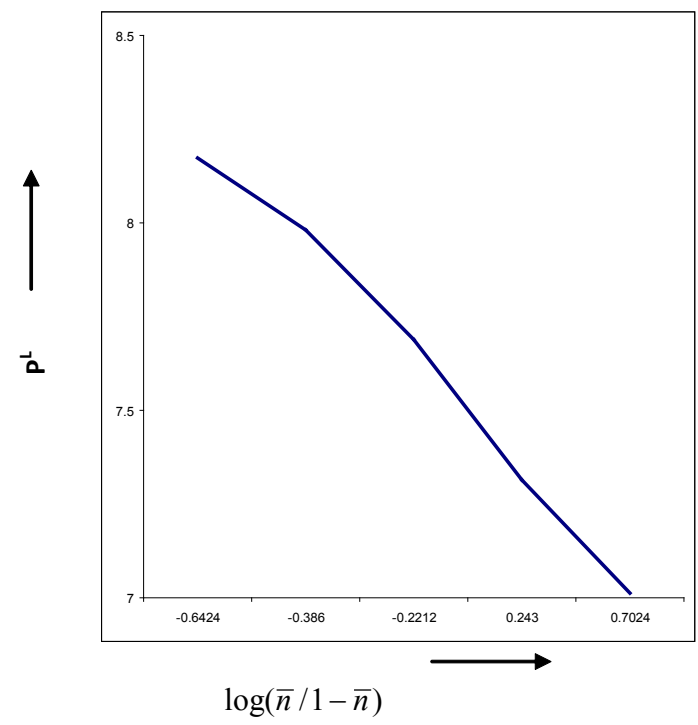

Fig.5d: Formation curve of $\mathrm{Zn}$ (II) Plot of $\log (\bar{n} / 1-\bar{n}) \mathrm{Vs} \mathrm{P}^{\mathrm{L}}$

Ligand: $\mathrm{HNNCl}\left(\mathrm{L}_{1}\right)$

Temp. $298 \pm 1 \mathrm{~K}$ $\mu^{\circ}=0.10(\mathrm{M}) \mathrm{KNO}_{3} \quad$ Water: Dioxane $=3: 2(\mathrm{v} / \mathrm{v})$ 


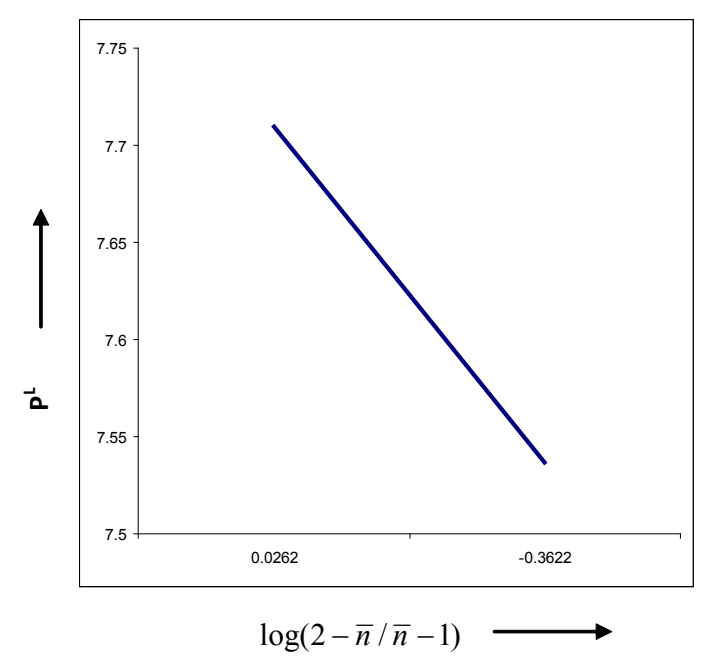

Ligand: $\mathrm{HNNCl}$

Temp. $298 \pm 1 \mathrm{~K}$ $\mu^{\circ}=0.10(\mathrm{M}) \mathrm{KNO}_{3} \quad$ Water: Dioxane $=3: 2(\mathrm{v} / \mathrm{v})$

Fig. 6a: Formation curve of $\mathrm{Co}(\mathrm{II})$ Plot of $\log (2-\bar{n} / \bar{n}-1)$ Vs PL

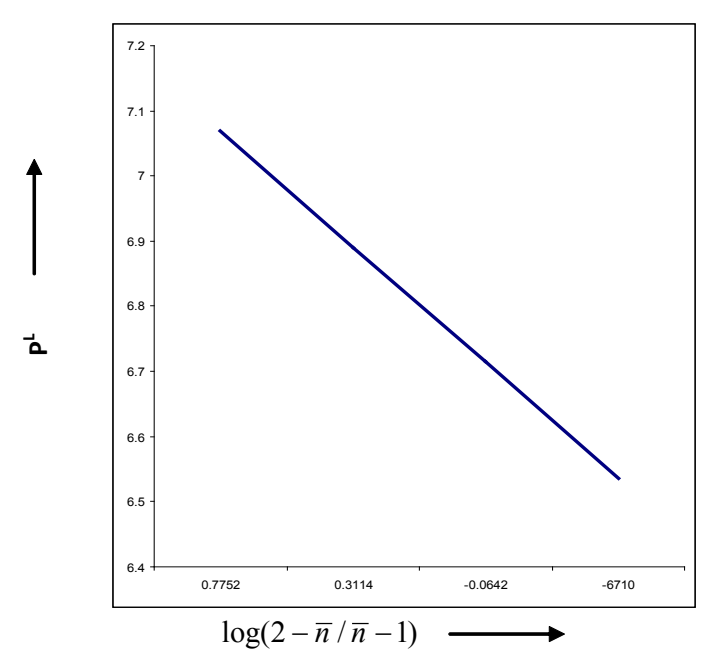

Ligand: $\mathrm{HNNCl}$ Temp. $298 \pm 1 \mathrm{~K}$

$\mu^{\circ}=0.10(\mathrm{M}) \mathrm{KNO}_{3}$

Water: Dioxane $=3: 2(\mathrm{v} / \mathrm{v})$

Fig.6c: Formation curve of $\mathrm{Cu}(\mathrm{II}) \mathrm{Plot}$ of $\log \left(2-\bar{n} /^{\bar{n}}-1\right) \mathrm{Vs} \mathrm{P} \mathrm{L}$

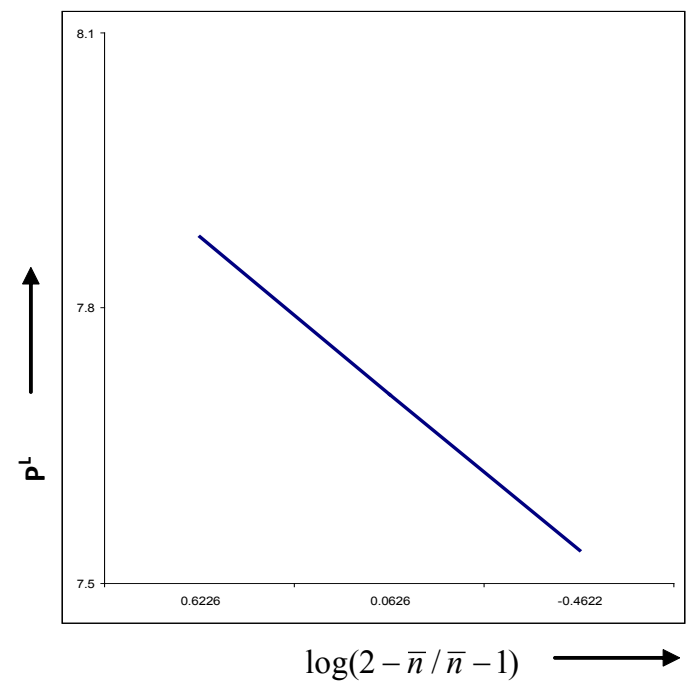

Ligand: $\mathrm{HNNCl}$

Temp. $298 \pm 1 \mathrm{~K}$ $\mu^{\circ}=0.10(\mathrm{M}) \mathrm{KNO}_{3} \quad$ Water: Dioxane $=3: 2(\mathrm{v} / \mathrm{v})$

Fig.6b: Formation curve of $\mathrm{Ni}(\mathrm{II})$ Plot of $\log \left(2-\bar{n} /^{\bar{n}-1)}\right.$ Vs PL

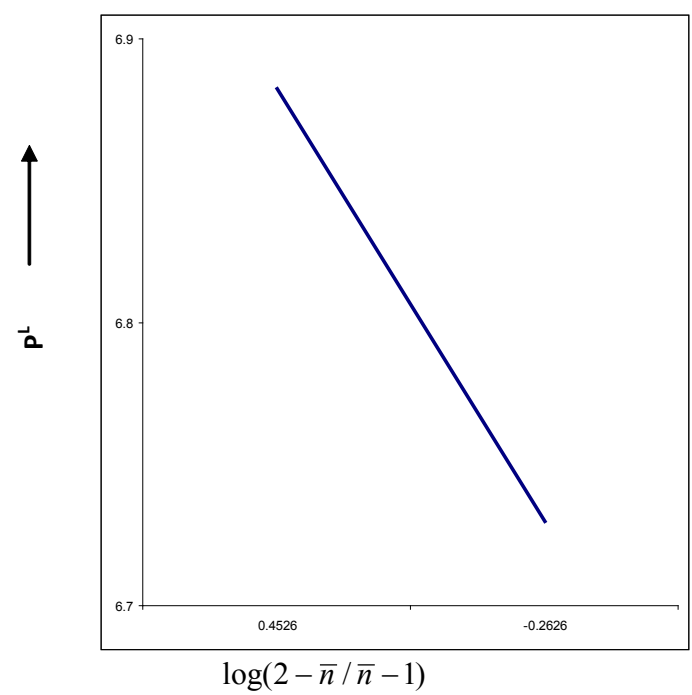

Ligand: $\mathrm{HNNCI}$ Temp. $298 \pm 1 \mathrm{~K}$ $\mu^{\circ}=0.10(\mathrm{M}) \mathrm{KNO}_{3} \quad$ Water: Dioxane $=3: 2(\mathrm{v} / \mathrm{v})$

Fig.6d: Formation curve of $\mathrm{Zn}$ (II)Plot of $\log \left(2-\left.\bar{n}\right|^{\bar{n}-1)}\right.$ Vs PL 
computational methods at temperatures $298 \mathrm{~K}$ are summarized in table no. 12

The different methods used are :-

a) Half - integral method

b) Mid - point calculation method

c) Straight line plot method.

The order of stability constant of various metals for the given ligand

$\mathrm{HNNCl}$ are $\quad-\quad \mathrm{Cu}(\mathrm{II})>\mathrm{Ni}(\mathrm{II})>\mathrm{Co}(\mathrm{II})>$ $\mathrm{Zn}(\mathrm{II})$

The values of stepwise stability constants and over all stability constants are given in table no. 13 For the given ligand the stability constants of metals show the sequence

$$
\mathrm{Cu}(\mathrm{II})>\mathrm{Ni}(\mathrm{II})>\mathrm{Co}(\mathrm{II})>\mathrm{Zn}(\mathrm{II})
$$

This is natural order given by Irving William. A theoretical justification of the order of stability constants follows from the consideration of the reciprocal of the ionic radii and $2^{\text {nd }}$ ionization enthalpy of metal. Calvin - Bjerrum titration technique modified by Irving and Rossotti was used to determine the practical proton ligand and metal ligand stability constants at constant ionic strength maintained by using dilute $\mathrm{KNO}_{3}$ solution. Irving and Rossotti pointed out that the formation constant of metal chelates can be obtained without converting the $\mathrm{pH}$ - meter reading $[\mathrm{B}]$ to stoichiometric hydrogen ion concentration and without knowing the stiochiometric concentration of neutral salts added to maintain ionic strength. This method is valid for both aqueous and non-aqueous medium.

The nitrate $\left(\mathrm{NO}^{-}\right)$ion has very slight complexing tendency. Therefore competition between nitrate ion and the ligand under study is of no importance.

The stability of the chelates is greatly affected by the electron density around the imino nitrogen ( $-\mathrm{C}=\mathrm{N}-$ ). Higher the electron density around the nitrogen atom, stronger is the metal ligand bond.

The difference between the successive stepwise stability constant is large, which suggest that the formation of $\mathrm{ML}$ and $\mathrm{ML}_{2}$ chelates take place. The results obtained are in conformity of our previous studies $^{12-15}$ and other workers ${ }^{16-17}$.

\section{REFERENCES}

1. Djebbar S. S.; Benali B.O.; Deloume J.P. Polyhedron, 1997, 16, 2175.

2. Bhattacharyya P.; Parr J.; Ross A. T., Chem. Soc. Dalton, 1998, 3149.

3. He L.; Gou S. H. ; Shi Q.F., J. Chem. Crystallography., 1999, 29207.

4. Wu J.C.; Tang N.: Liu W.S.; Tan M.Y. ; Chan A.S., Chin Chem. Lett., 2001, 12757.

5. Liu C. M. ; Xiong R.G.; You X. Z.; Liu Y.J.; Cheung K.K., Polyhedron, 15 1996, 45651.
6. Djebbar S.S.; Benali B.O.; Deloume J.P., Transit Metal Chem., 1998, 23, 443.

7. Hamada Y.J., IEEE Trans. Electron Devices, 1997, 44, 1208.

8. Brown D. H., Smith W.E., Teape J.W. et al., J. Med. Chem., 1980, 23(7), 729.

9. Srivastava R.S., Ind. J. Chem., 1990, 29 , 1024 - 1026.

10. Waish C., Nature, 2001, 409, 226. 